\title{
Trivium
}

Revue franco-allemande de sciences humaines et sociales - Deutsch-französische Zeitschrift für Geistesund Sozialwissenschaften

30 | 2019

La constitution au tournant des XXe et XXle siècles

\section{Verfassung und Konstitutionalismus}

\section{Olivier Beaud}

Traducteur : Olivier Joop

\section{(2) OpenEdition}

\section{Journals}

\section{Édition électronique}

URL : http://journals.openedition.org/trivium/6584

DOI : $10.4000 /$ trivium.6584

ISSN : 1963-1820

Éditeur

Les éditions de la Maison des sciences de l'Homme

\section{Référence électronique}

Olivier Beaud, "Verfassung und Konstitutionalismus », Trivium [Online], 30 | 2019, online erschienen am 18 Dezember 2019, abgerufen am 08 September 2020. URL : http://journals.openedition.org/ trivium/6584; DOI : https://doi.org/10.4000/trivium.6584

Ce document a été généré automatiquement le 8 septembre 2020

\section{(c) (i) () $\Theta$}

Les contenus des la revue Trivium sont mis à disposition selon les termes de la Licence Creative Commons Attribution - Pas d'Utilisation Commerciale - Pas de Modification 4.0 International. 


\title{
Verfassung und Konstitutionalismus
}

\author{
Olivier Beaud \\ Traduction : Olivier Joop
}

\section{NOTE DE L'ÉDITEUR}

Wir danken Herrn Olivier Beaud und dem Verlag Presses universitaires de France für die freundliche Genehmigung, diesen Artikel in deutscher Übersetzung zu publizieren. Nous remercions M. Olivier Beaud et les Presses universitaires de France de nous avoir accordé l'autorisation de traduire ce texte pour le présent numéro.

»Als Konsequenz dieser Entwicklung [der Rechtsauffassung]«, schreibt Giovanni Sartori,

»muß man heute jede Art staatlicher Organisation als >Verfassung bezeichnen und jede Art staatlicher Verfügung, die die vom Souverän festgelegte Form aufweist, als `Gesetzı. [...] Nach der formalen Auffassung kann das Recht jeden beliebigen Inhalt haben, und ein ungerechtes Recht ist dennoch Recht. Daher kann die Gesetzgebung offen tyrannisch sein und trotzdem nicht nur legal heißen, sondern auch als rechtmäßig zu achten sein. $\aleph^{1}$

So verstanden, enthielte der moderne Verfassungsbegriff eine offensichtliche Diskrepanz zwischen Legalität und Legitimität, insofern er auch eine Verfassung einschlösse, die sich als unterdrückerisch und sogar tyrannisch entpuppen könnte. Aus Sicht des Verfassungspositivismus, der die (politische und juristische) Wirklichkeit mit den Mitteln der Wissenschaft untersuchen will, muss jeder Rechtstext, der in Form eines Verfassungsgesetzes verabschiedet worden ist, auch Verfassung genannt werden. Für den Konstitutionalismus hingegen, der sich den Schutz der Freiheit des Einzelnen mittels Verfassungsnormen auf die Fahnen geschrieben hat, muss eine wirkliche Verfassung auch gerecht - oder, um einen moderneren Begriff $\mathrm{zu}$ verwenden, freiheitlich-demokratisch - sein, was bedeutet, dass sie übergeordnete Grundsätze wie die Menschenrechte und die Gewaltenteilung würdigt, welche die politische Macht begrenzen sollen. Auf begrifflicher Ebene könnte es somit zu einem Konflikt kommen zwischen dem Konstitutionalismus und einem im Sinne des Verfassungspositivismus 
verstandenen Verfassungsbegriffs. Auf den ersten Blick könnte dieser Konflikt als Wiederkehr in neuem Gewand des klassischen und jahrhundertalten Ringens der Theorie vom Naturrecht und der Theorie vom Rechtspositivismus gesehen werden. Träfe diese Annahme zu, genügte es allerdings, in diesem Lexikon die Einträge »Recht« (Naturrecht, positives Recht) oder "Alte und Neue" (Anciens et Modernes) nachzuschlagen, um eine Antwort auf die Frage des Verhältnisses von Verfassung und Konstitutionalismus zu erhalten.

2 In diesem Eintrag wird jedoch die Ansicht vertreten, dass mit der Naturrechtsproblematik das Verhältnis zwischen Verfassung und Konstitutionalismus nicht erschöpfend beschrieben wird. Zum einen, weil die Begriffe Verfassung und Konstitutionalismus bei weitem nicht eindeutig sind und ihre Bedeutung näher bestimmt werden muss, bevor sie verglichen werden können. Zum anderen, weil im Vorhinein die Frage der Entsprechung von Verfassung und Recht zu klären ist, die der Verfassungspositivismus postuliert - eine Gleichsetzung, die sich bei einer eingehenden Analyse des Verfassungsbegriffs nicht so ohne weiteres ergibt.

\section{Der Begriff der Verfassung und der Begriff des Konstitutionalismus}

3 Ursprünglich findet der aus dem Lateinischen constitutio stammende Begriff der Konstitution, bzw. Verfassung, Verwendung sowohl im Bereich der Medizin (wo er den Zustand, die Ordnung oder die Struktur einer Sache beschreibt) als auch im Recht, als Bezeichnung für eine Sammlung päpstlicher oder klösterlicher Rechtstexte und eine Art öffentlicher Urkunde. Darüber hinaus kann er sowohl auf den Körper eines Menschen (»menschliche Konstitution«) als auch auf ein soziales oder abstraktes Gebilde angewendet werden. Die reichhaltige Bedeutungsvielfalt des Begriffs hat $\mathrm{zu}$ einer ebenso breitgefächerten Verwendung geführt. Die Idee der Verfassung ihrerseits ist vom scharfen Gegensatz durchdrungen zwischen zwei Vorstellungen, die hier als institutionelle (Bobbio) und normative Auffassung der Verfassungsidee bezeichnet werden können.

4 Nach der institutionellen oder auch »organischen« Auffassung regelt die Verfassung die politische "Ordnung " oder ist sie das »oberste Prinzip der politischen Einheit bzw. der politischen Ordnung" (Fioravanti). Als Struktur regelt sie die Handlungen und die Daseinsweise des Staates auf die gleiche Weise wie die Konstitution das Leben und die Bewegungen des menschlichen Körpers bestimmt. Daraus folgt, dass jeder Staat eine Verfassung besitzt, denn »alles, was existiert, hat auch eine Daseinsform, gleich ob gut oder schlecht, vernünftig oder nicht« (P. Rossi). Sehr oft geht diese Vorstellung von der Verfassung einher mit illiberalen politischen Überzeugungen, denn dieser Vorrang der politischen Ordnung - des Ganzen - setzt voraus, dass es eine (oder mehrere) Gewalt(en) gibt, die in der Lage ist (bzw. sind), eine solche Ordnung zu erschaffen und aufrechtzuerhalten. Die Verfassung in diesem Sinne ist es dann, die die Einheit eines Volkes gegenüber den (innerstaatlichen oder äußeren) Zentrifugalkräften bewahrt, die diese Einheit ständig bedrohen. Als maßgebliche politische Denker in dieser Traditionslinie sind Hobbes, Hegel und Carl Schmitt zu nennen. Freilich folgen auch andere politische Philosophen, wie Aristoteles oder Montesquieu, der institutionellen Auffassung von Verfassung, ohne dabei gleichzeitig auch dieses Ordnungsschema zu verfechten. Auf der anderen Seite betrachtet die normative Auffassung der 
Verfassungsidee die Verfassung als Grundgesetz, mit anderen Worten als oberste Rechtsnorm. Sie reiht sich ein in die Denktradition Lockes, Constants oder auch Rawls', die in der Verfassung ein Instrument zur Begrenzung der Macht zum Zwecke des Schutzes der Freiheit des Einzelnen erblickt.

5 Die Idee des Konstitutionalismus ist nicht minder mehrdeutig als diejenige der Verfassung. Im weitesten Sinne beschreibt das Konzept des Konstitutionalismus das Verfahren, »durch Gewaltenteilung (Aufteilung der Macht) ein System wirksamer Beschränkungen für das Handeln der Regierung " zu schaffen. ${ }^{2}$ So verstanden, verdichtet der Konstitutionalismus zwei entscheidende und bereits weit zurückreichende Gedanken der politischen Philosophie: zum einen die Forderung nach einer begrenzten Herrschaft und zum anderen die Herrschaft des Rechts, die an die Stelle der Herrschaft der Menschen tritt. Nach einer solchen Sichtweise vermöchte der Konstitutionalismus die Einschränkung der Macht sowohl der Polis (»antiker Konstitutionalismus«) als auch der Macht des Königtums durch Gewohnheitsrecht (»mittelalterlicher Konstitutionalismus«) zu erklären. Im engeren Sinne hingegen bezeichnet Konstitutionalismus zwar die Beschränkung politischer Herrschaft, doch ist diese politische Gewalt ausschließlich diejenige des modernen Staates. Der Konstitutionalismus ist in der Tat integraler Bestandteil der Idee einer freiheitlichen Demokratie, die eine Unterscheidung zwischen privater oder auch sozialer Sphäre einerseits und öffentlichem oder auch politischem Raum andererseits voraussetzt, mit anderen Worten eine Abgrenzung von Staat und Bürger- bzw. Zivilgesellschaft, die den früheren Denkformen des Konstitutionalismus unbekannt war.

6 Hier soll nur der Konstitutionalismus im engeren Sinne behandelt werden, da der antike und der mittelalterliche Konstitutionalismus mit der Entstehung der Idee der Souveränität und des modernen Staates überholt sind. Der Konstitutionalismus geht vom Bestehen eines Gefüges unantastbarer und die "Verfassung « bildender Normen aus. Entgegen der Ansicht Mac Ilwains hat die Entstehung des modernen (und souveränen) Staatswesens den mittelalterlichen Konstitutionalismus vollständig verdrängt, denn durch das Konzept der Souveränität kann der Souverän auch über Normen nicht-staatlichen Ursprungs, wie etwa das Gewohnheitsrecht, verfügen. Anders ausgedrückt, kann der souveräne Staat jede Norm des positiven Rechts (also auch die Regeln des Gewohnheitsrechts, die nach der mittelalterlichen Auffassung die "Verfassung" bildeten) nach seinem Belieben ändern, auch aus politischen Erwägungen. Diese Feststellung entkräftet freilich nicht die Idee eines modernen Konstitutionalismus, und zwar aus dem Grund, dass sich dieser innerhalb des Staates gewissermaßen durch einen involutiven Prozess entwickelt. Die Souveränität des Staates ist also Teil der modernen Idee von Konstitutionalismus. Dieser ist bestrebt, die Staatsgewalt mittels "unantastbarer" und "Verfassungsnormen" genannter Vorschriften einzuhegen, die außerhalb der Verfügungsgewalt der Herrschenden stehen. Genauer gesagt: Die Entstehung der modernen Verfassung ist Ausdruck des Bestrebens, zum Schutze der Rechte der Bürger einen Teil des positiven Rechts der Verfügungsmacht der Herrschenden zu entziehen. Der Konstitutionalismus ist untrennbar mit John Locke, dem zentralen Denker des Konstitutionalismus, und dessen Begriff des Vertrauens (»trust«) verbunden, gemäß welchem das zum Souverän gewordene Volk (als »community" bezeichnet) der Regierung sein Vertrauen gibt und sie überwacht, um sicherzustellen, dass die Rechte der Bürger gewahrt bleiben. Eingebettet in diese Beziehung zwischen Menschenrechten und Souveränität des Volkes, folgt der Konstitutionalismus dem Locke'schen Programm: „So hielt das Volk klare Grenzen der 
Prärogative für notwendig in Fällen, die es [...] der Weisheit solcher Fürsten überlassen hatten $[. ..] . \ll^{3}$ Historisch betrachtet, besteht der Sieg des Konstitutionalismus vorrangig darin, die Prärogative bzw. das »Vorrecht« des Königs, mit anderen Worten die absolute Macht des Herrschers, zurückgedrängt zu haben. Es darf damit behauptet werden, dass seit der Zeit Lockes die fundamentale Trennlinie im Bereich des Staatsaufbaus zwischen der absoluten, als willkürlich bezeichneten Macht und der begrenzten, als verfassungsmäßig betitelten Gewalt verläuft. Im Verfassungsstaat sind die Regierenden durch das Recht gebunden, das sie gleichzeitig gegen den Missbrauch der Staatsmacht schützt. Der Konstitutionalismus schließt die Monarchie als Staatsform nicht aus und kann eine in Frankreich als »begrenzte Monarchie» (S. Rials), allgemein als »konstitutionelle Monarchie« bezeichnete Staatsorganisation beinhalten. Die Frage der Regierungsform (Monarchie oder Republik) ist für den Konstitutionalismus nachrangig, entscheidend sind dessen Grundsätze und Wirkmechanismen.

7 Die Ursprünge des Konstitutionalismus sind unzweifelhaft in der Philosophie des politischen Liberalismus zu finden, jedoch besteht seine Besonderheit darin, dass er die von ihm angestrebte Begrenzung politischer Macht mittels des Rechts zu erreichen sucht, durch eine als Rechtsordnung verstandene Verfassung. In dieser Hinsicht unterscheidet sich der moderne Konstitutionalismus von der altgriechischen (Verfassung als Ordnung) und der mittelalterlichen (Verfassung als Gewohnheitsrecht) Verfassungsvorstellung. Da die Entwicklung des modernen Rechts selbst in Richtung verbindlicher Rechtsnormen tendiert, strebt auch der moderne Konstitutionalismus in Richtung einer Verfassung als Rechtsnorm, wie dies die gängigen Definitionen des Verfassungsbegriffs aufzeigen. "Die moderne Verfassung zeichnet sich", im gebräuchlichen (d.h. normativen) Sinne verstanden, »durch den Anspruch aus, politische Herrschaft nach Zustandekommen und Ausübungsweisen in einem allen anderen Rechtsnormen übergeordneten Gesetz umfassend und einheitlich zu regeln. $\ll^{4}$ Aus dieser Definition ergeben sich vier grundlegende Wesensmerkmale der als Rechtsnorm verstandenen Verfassung.

8 Gemäß dem ersten dieser Merkmale bestimmt und gestaltet die Verfassung die Übertragung und Ausübung der staatlichen öffentlichen Gewalt. Sie ermächtigt die Regierenden, zu handeln, indem sie deren Befugnisse, die dadurch zu Zuständigkeiten werden, festlegt und damit auch gleichzeitig eingrenzt. Rechtlich ist die Verfassung mehr Ermächtigung als Anordnung. Der zweite Wesenszug der Verfassung liegt in ihrer Schutzfunktion der Rechte des Einzelnen gegen einen möglichen Missbrauch der Staatsgewalt. Hier zeigt sich ihre originär liberale Komponente, die den Gesichtspunkt der natürlichen Rechte des Menschen mit dem Gedanken der Einschränkung der Macht der Herrschenden verbindet. Der dritte Aspekt besteht in der die Ausübung der Staatsgewalt eingrenzenden Funktion der Verfassung, eine Begrenzung, die durch den Grundsatz der Gewaltenteilung, d. h. durch die Aufteilung der von den handelnden öffentlichen Gewalten ausgeübten Befugnisse auf unterschiedliche Träger, gewährleistet wird. Bekanntermaßen fasst Artikel 16 der Erklärung der Menschen- und Bürgerrechte von 1789 die beiden letztgenannten Charakteristika zusammen, wenn er verkündet: »Eine Gesellschaft, in der die Verbürgung der Rechte nicht gesichert und die Gewaltenteilung nicht festgelegt ist, hat keine Verfassung." Das letzte Wesensmerkmal einer modernen Verfassung ist schließlich, dass es sich bei ihr um ein formal höchstes, allen anderen Rechtsvorschriften vorgehendes Gesetz handelt, das in einem einzigen und besonderen Dokument niedergelegt ist. In der Tat ist die 
Verfassung mit wenigen Ausnahmen, wie beispielsweise Großbritannien oder Israel, ein schriftlich festgehaltenes Gesetz, eine Art Verfassungsgesetzbuch. ${ }^{5}$

\section{Konstitutionalismus und Verfassung als Rechtsnorm: der englische, amerikanische und französische Konstitutionalismus}

9 Im Zuge der Amerikanischen und der Französischen Revolution am Ende des 18. Jahrhunderts nimmt der Konstitutionalismus eine normative Auffassung von Verfassung an. Es kommt zu einem Paradigmenwechsel in Bezug auf die Verfassung, wie eine Gegenüberstellung des Konstitutionalismus nach Bolingbroke und des von den amerikanischen und französischen Revolutionären (Thomas Paine und sieyès) vertretenen Konstitutionalismus veranschaulicht.

\section{Auf Gewohnheitsrecht gegründete Verfassung und englischer Konstitutionalismus}

1733 schlägt Bolingbroke, bekannt als Vordenker einer ausgeglichenen Gewaltenteilung, die folgende Begriffsbestimmung für die Verfassung vor: »Unter Verfassung verstehen wir, wenn wir uns genau und angemessen ausdrücken, dieses Gefüge von Gesetzen, Institutionen und Sitten, die aus bestimmten unveränderlichen Grundsätzen der Vernunft folgen, auf bestimmte, unveränderliche Aspekte des Gemeinwohls hinwirken und zusammen die allgemeine Herrschaftsform bilden, auf die sich die Gemeinschaft zu seiner Regierung geeinigt hat «. ${ }^{6}$ Diese Betrachtungsweise, die man als politische Auffassung von Verfassung bezeichnen könnte, verbindet die klassische Sichtweise von Verfassung als Organisation von Herrschaft mit der englischen und gewohnheitsrechtlichen Vorstellung des Verfassungsbegriffs. Das Erbe des klassisch-antiken politischen Denkens besteht darin, die Verfassung als Regierungsform, als politische Ordnung zu begreifen (die politeia/ $\pi$ o $\lambda \imath \tau \varepsilon i ́ \alpha$ der Griechen). In diesem Punkt will Bolingbroke an das von Cicero propagierte Ideal der Mischform von Regierung ${ }^{7}$ anknüpfen, indem er das Gleichgewicht zwischen den die englische Verfassung bildenden Organen als vorbildhaft rühmt. Hier treten die vier Elemente der republikanischen constitutio erneut zum Vorschein: Autorität durch Alter, Gleichgewicht durch Austarieren der politischen und gesellschaftlichen Kräfte, Aufgabe und Pflicht, die Freiheit der Bürger zu schützen, und schließlich Dauerhaftigkeit und Beständigkeit. ${ }^{8}$ Der zweite Ursprung dieser Auffassung von Verfassung liegt in der englischen Verfassungspraxis. Die englische Regierungsform kennt zwar das Gleichgewicht der Kräfte, agiert aber im Wesentlichen auf der Grundlage der Vernunft und einer Reihe von in den Institutionen der englischen Nation verinnerlichten Grundsätzen, die als »Verfassung« bezeichnet werden. Das Wesensmerkmal dieser Verfassung ist, dass sie die Macht der Krone nicht durch ein geschriebenes Gesetz, sondern durch Privilegien und Rechte einschränkt, die aus von den Gerichten anerkannten Gewohnheiten entstanden sind. In dieser Art von Verfassung, die auf Gewohnheitsrecht basiert, stellt der rechtliche Präzedenzfall das lebensnotwendige Prinzip für die Staatsorgane und die Gesetzgebung dar. Es ist diese Tradition, auf die 
sich Burke bezog, als er die englischen Verfassungsgrundsätze gegen die Französische Revolution verteidigte.

\section{Amerikanischer Konstitutionalismus}

11 Auf den Konstitutionalismus amerikanischer Prägung geht die geschriebene Verfassung zurück. Als Gegenmodell zur englischen Gewohnheitsrechtsverfassung steht der moderne Konstitutionalismus, der unter Verfassung eine schriftliche Verfassung versteht. In diesem neuen Bedeutungsgehalt des Wortes kommt ein fundamentaler Wandel zum Ausdruck: die »Verrechtlichung" der Verfassung durch die Verschmelzung des Verfassungsbegriffs mit dem Begriff des »Grundgesetzes«. Diese einschneidende Entwicklung nimmt ihren Ursprung in den Vereinigten Staaten. Der Freiheitskämpfer Thomas Paine, erbitterter Gegner der Theorien Burkes, steht exemplarisch für diese neue Strömung im staatsrechtlichen Denken, die in der Verfassung einen rechtlichen Begriff sieht. Die Verfassung wird definiert als »Werk [nicht] der Regierung, sondern des Volkes, das eine Regierung einsetzt«. Thomas Paine führt weiter aus: »Eine Konstitution ist ein Etwas, das der Regierung vorherging; und die Regierung ist nur das Geschöpf der Konstitution. «9 Aus der Tatsache, dass die Verfassung der Regierung vorhergeht, ergibt sich, dass die einzig legitimen Gewalten in einer verfassungsmäßigen Demokratie diejenigen Gewalten sind, die von der Verfassung eingerichtet wurden (in Frankreich werden diese mit dem Begriff "Verfassungsorgane" [pouvoirs publics] bezeichnet), und dass die Verfassung die gegenüber allen anderen von den Regierenden erlassenen Rechtsakten vorrangige Rechtsnorm darstellt. Sie ist ein "grundlegendes Gesetz«. Woher kommt diese Verschmelzung von Verfassung und »Grundgesetz« und welche Tragweite hat sie?

Die Bedeutung des »Grundgesetz«-Begriffs liegt darin, dass er eine Abgrenzung der unantastbaren Normen (»Verfassungsnormen« lato sensu), d.h. die der Verfügungsgewalt des Souveräns entzogen sind, gegenüber den nicht unantastbaren Vorschriften, die durch den Willen der Regierenden geändert werden können, ermöglicht. Allerdings stand dieser Begriff nicht immer im Dienste der Verfassungsidee. Ursprünglich verwendeten ihn die Rechtsgelehrten zu Zeiten der französischen Monarchie in der Pluralform und in einem einschränkenden Sinne: die Grundgesetze stellten das öffentliche Recht des Königreiches dar, von dem der Monarch nicht abweichen durfte, da es den Staat gegen mögliche Verirrungen des Mannes auf dem Thron schützen sollte. In diesem Sinne ist Bodins Wendung von der Unveräußerlichkeit der Güter der Krone zu verstehen, und in demselben Sinne verwendeten auch Jakob I. und Francis Bacon den Begriff, wenn sie damit die "unveräußerlichen Vorrechte der Krone«, das Ius Regis, im Gegensatz zum gemeinen Recht, dem common law, bezeichneten. Der Bedeutungsgehalt der Wendung "Grundgesetze« erfuhr jedoch eine Umkehrung in einen freiheitlichen Sinn, als die Verteidiger des englischen Parlaments - die von den französischen, hugenottischen Autoren der Zeit der Religionskriege beförderte Verwendung des Begriffes aufgreifend - mit diesem Begriff die Gesamtheit aller wesentlichen Rechte der Untertanen bezeichneten, die der König nicht abschaffen dürfe. Seit dieser Zeit wird der Gedanke der "Grundgesetze« geltend gemacht, wenn es um den Schutz der Freiheiten und Vorrechte der »Bürger« oder der verschiedenen gesellschaftlichen Gruppierungen geht (J. Gough). 
13 Der letzte qualitative Sprung erfolgte, als der Ausdruck »Grundgesetz« eine spezifisch rechtliche Bedeutung im Sinne eines obersten Gesetzes annahm und mit der Verfassungsidee verknüpft wurde. Dieses Ereignis geschah in Amerika im Zuge der Unabhängigkeitsbewegung, die zur Amerikanischen Revolution führen sollte. In deren Verlauf kam es zur Geburt der modernen Verfassung als oberster Rechtsnorm eines Staates, $d . h$. als gegenüber allen anderen Normen vorrangiger Bestimmung, welche die Ungültigkeit oder Nichtigerklärung von Vorschriften erwirken kann, die mit ihr unvereinbar sind. Zwei Begebenheiten sind für diese Entwicklung ursächlich. Zum ersten trat an die Stelle des Widerstandsrechts die Möglichkeit, gerichtlich gegen einen verfassungswidrigen Akt vorzugehen, was zu einer Befriedung der politischen Auseinandersetzungen durch das Recht führte. Während das englische Verfassungsrecht sich als unfähig erwies, auf ein verfassungswidriges Gesetz zu reagieren, und somit nur das Widerstandsrecht Abhilfe leisten konnte, schuf das amerikanische Verfassungsrecht neue Lösungsmöglichkeiten für politische Konflikte, nämlich mit friedlichen Mitteln und durch ausgestaltete Verfahren: insbesondere durch die Möglichkeit einer gerichtlichen Prüfung der Verfassungsmäßigkeit von Gesetzen, aber auch durch andere Verfahren wie beispielsweise die Bildung vorübergehender verfassungsgebender Versammlungen (die berühmten "Verfassungskonvente«) oder die Ausgestaltung, in den Verfassungstexten selbst, von Verfahren zur Änderung der Bestimmungen der Verfassung. Das zweite bedeutende Ereignis war die Entstehung einer schriftlichen, sich ausdrücklich von den einfachen Gesetzen abhebenden Verfassung. Dadurch erhielt die Verfassung Rechtsverbindlichkeit, entfaltete also eine Bindungswirkung gegenüber den Regierenden. Diese begriffliche Umwälzung ermöglichte die feste Verankerung des Schutzes der Menschenrechte durch die Schaffung eines entsprechenden Rechtwegs. Die Verbindung der Verfassung mit der Möglichkeit einer Verfassungswidrigkeitsklage als Abhilfe gegen den Missbrauch der Staatsgewalt vollzog der Oberste Richter John Marshall im vom Obersten Gerichtshof der Vereinigten Staaten im Jahr 1803 entschiedenen Fall Marbury v. Madison. In diesem Urteil beschrieb er die moderne Rechtsauffassung einer Verfassung:

"All diejenigen, die schriftliche Verfassungen ausgearbeitet haben, betrachten diese als grundlegendes und oberstes Gesetz der Nation, daher muss für jede Regierungsform, die auf einer solchen Verfassung beruht, gelten, dass Gesetze, die gegen die Verfassung verstoßen, unrechtmäßig sind.»

Ein derart verstandener Verfassungsbegriff setzt freilich den Gedanken an Rangunterschiede zwischen den Rechtsnormen voraus, was seit Kelsen als Stufenbau der Rechtsordnung oder auch Normenhierarchie bezeichnet wird. Der niedrigere Rang des einfachen Gesetzes gegenüber dem Verfassungsgesetz oder, in Bezug auf die Staatsorgane, die Unterordnung des Parlaments gegenüber dem Verfassungsgeber, ist »die große, von der Amerikanischen Revolution bewirkte Neuerung ${ }^{10}{ }^{10}$

\section{Französischer Konstitutionalismus}

14 Die Französische Revolution scheiterte zwar in Bezug auf die praktische Anwendung der Verfassungsnorm, gab Europa dafür allerdings die abstrakteste Vorstellung des neuen Verfassungsparadigmas. Abstrakte Vorstellung deshalb, weil die Verfassung auf philosophischen Grundsätzen beruht, die in der Erklärung der Menschen- und Bürgerrechte auf erhabene Weise niedergelegt sind: sie soll die natürlichen Rechte des 
Menschen zum "positiven Recht erheben«. Diesbezüglich ist das Werk von Emmanuel Sieyès umfassend, mehr noch vermutlich als dasjenige von Condorcet. Während die Mitglieder des Parlaments in Kategorien überlieferter Rechte dachten, beschwört Sieyès, im Geiste der Aufklärung und der Physiokraten, aus der Natur und der Vernunft abgeleitete Rechte. Im vollen Vertrauen auf die neue »Verfassungswissenschaft« will er die Entstehung einer schriftlichen Verfassung erreichen, welche die Vorgaben der abstrakten Vernunft (als wahrhaftiges »Verfassungsgesetzbuch«) und die rechtlich zum Schutz der Menschenrechte notwendigen Maßnahmen miteinander in Einklang bringen soll.

15 In Was ist der Dritte Stand? gelingt es ihm, die scheinbar widersprüchlichen Ideen der Souveränität der Nation auf der einen und der Verfassung auf der anderen Seite zusammenzuführen. Durch den von ihm formulierten Gedanken der Souveränität und der verfassungsgebenden Gewalt rechtfertigt er das Recht auf Widerstand gegen Unterdrückung, das dem Volk zustehe, wenn die Regierung die wahrhaftige Verfassung verletze. Damit markiert sein Werk sichtbar den Bruch mit dem klassischen politischen Denken, den Ideen Bolingbrokes, nach dem die Nichteinhaltung der Verfassung die Regierung disqualifiziert - sie ist dann eine schlechte Regierung -, aber keine konkreten politischen Folgen nach sich zieht. Sieyès begnügt sich jedoch nicht damit, das Recht zum Aufstand zu verteidigen, er entwickelt auch ein Modell für die Funktionsweise einer Verfassung. Er führt das klassische Verständnis der Verfassung als Anordnung oder Gliederung der Gewalten (politeia) und den modernen Begriff der Verfassung als Grundnorm zusammen.

Auf der einen Seite gibt er die strukturbildende Eigenschaft der Verfassung durch das organische Bild des "politischen Körpers« bzw. der "politischen Körperschaft" (corps politique) wieder. So ist es ihm möglich, Assoziationen zu nutzen, die diese medizinischbiologische Metapher mit sich bringt. »Man kann unmöglich eine Körperschaft zu einem bestimmten Zweck schaffen, ohne ihr eine Organisation, Formen und Gesetze zu geben, durch die sie in der Lage ist, die ihr gestellten Aufgaben zu erfüllen. Man nennt dies die Verfassung dieser Körperschaft. ${ }^{11}$ Auf der anderen Seite stellt er jedoch fest, dass, wenn die Errichtung einer »Regierung" notwendig dafür sei, damit der Staat bestehen und wie eine politische Gewalt handeln könne, so müsse sie gleichzeitig für den Einzelnen nutzbringend sein und ihm mit Wohlwollen begegnen. Sieyès führt aus, es liege im Interesse der Nation,

»dass die übertragene Staatsgewalt niemals für ihre Auftraggeber schädlich werden darf. Daher die vielen politischen Vorsichtsmaßregeln, die man mit der Verfassung verwoben hat, die gleichzeitig unabdingbare Vorschriften für die Regierung sind, ohne die die Ausübung der Staatsgewalt ungesetzlich wäre. «12

Dies ist die zweite freiheitliche Dimension der Verfassung, die nunmehr als grundlegendes Gesetz betrachtet wird. Ebenso wie bei den amerikanischen Verfassungsvätern wird sie als rechtliches Mittel zum Schutze der Souveränität des Volkes gegen die Regierenden verstanden, da sie die Unterordnung der verfassten Gewalten (Staatsorgane) unter die verfassungsgebende Gewalt (Nation) impliziert. Sieyès gelingt es, die hergebrachte Idee der Verfassung als Regierungsform und den modernen Gedanken einer obersten verfassungsmäßigen Rechtsnorm miteinander zu vereinbaren.

17 Darüber hinaus hat er die entscheidende Bedeutung der Verfahren zur Sicherung der Verfassungsnorm erfasst, wie seine Befürwortung der Einrichtung einer Verfassungsgerichtsbarkeit (ausgeübt durch eine »Jury constitutionnaire«) belegt. Aber 
genau an diesem Punkt des Schutzes der Verfassung (Schutz der Menschenrechte) und ihrer rechtlichen Wirksamkeit liegt eine erstaunliche Diskrepanz zwischen der Verfassungsphilosophie einiger Revolutionäre und der Verfassungswirklichkeit während der Französischen Revolution. Tatsächlich ist der Konstitutionalismus in jener Zeit infiziert von der Form von Heiligkeit, die dem Gesetz als »Ausdruck des allgemeinen Willens« (Art. 6 der Erklärung von 1789) vom »Legizentrismus« (S. Rials) zugeschrieben wird, der dem Gesetz ein übersteigertes Vertrauen entgegenbringt, wenn es um den Schutz der Grundrechte geht. In einer Zeit politischer Wirren und Umwälzungen bleiben die von der Aufklärung geleiteten Bemühungen einiger girondistischer Abgeordneter und Condorcets (der die Bürger zu Verfassungswächtern erheben will), Mechanismen zum Schutz der Verfassung institutionell zu verankern, erfolglos, sodass die Verfassung »eine Vorrichtung, die für machtpolitische Zwecke verwendet wird, bleibt « und nicht »die Rechtsstellung eines obersten Gesetzes « hat, ${ }^{13}$ die sie in den Vereinigten Staaten erlangen konnte.

Die Analyse der Amerikanischen und der Französischen Revolution zeigt, dass es ihnen gelang, vormals unverbundene politische und rechtliche Auffassungen des Verfassungsgedankens zusammenzuführen. Die Verfassung wird zum Rechtsbegriff, indem sie zwischen gesetzlich und ungesetzlich unterscheidet, aber sie ist auch ein politisches Konzept, das die Staatsgewalt strukturiert und begrenzt. Mit den Worten Niklas Luhmanns: "Man denkt jetzt bei constitution an einen Rechtstext, der zugleich die politische Konstitution eines Staates fixiert $\ll \cdot{ }^{14}$ Auf diese Weise werden Recht und Politik, Verfassung und Konstitutionalismus vereint. Allerdings wird noch zu sehen sein, dass dieses Zusammentreffen von Konstitutionalismus und Verfassung auch wieder in Frage gestellt worden ist.

\section{Die Auflösung der Verbindung zwischen Verfassung und Konstitutionalismus nach der Französischen Revolution}

19 Nach dem "Verfassungslabor", das die Französische Revolution dargestellt hatte und das sowohl von den Jakobinern als auch vom Bonapartismus maßgeblich mitgeprägt wurde, musste sich der Konstitutionalismus in Europa einer doppelten Herausforderung stellen: Verfassung und Souveränität des Volkes miteinander in Einklang zu bringen und sich des Frontalangriffs Hegels zu erwehren, der gegen den Konstitutionalismus der Revolutionszeit konterte. Beiden Herausforderungen ist gemein, dass sie eine Entzweiung von Verfassung und Konstitutionalismus bewirken.

\section{Benjamin Constant oder die Trennung zwischen Verfassung als Rechtsnorm und Konstitutionalismus}

Der Konstitutionalismus gewinnt mit der Herausbildung der Demokratie eine zusätzliche Funktion: Kampf gegen die Tyrannei der Mehrheit. Das Problem der Französischen Revolution war der potenzielle Gegensatz zwischen der Souveränität des Volkes und der Freiheit des Volkes gewesen: Wie konnte verhindert werden, dass diejenigen, die sich auf die Souveränität des Volkes beriefen, diese an sich rissen? Dieses Problem, welches das Denken der amerikanischen Aufständischen (siehe die 
Federalist Papers) beherrscht hatte, wurde zwar von einigen scharfsichtigen Protagonisten der Französischen Revolution erkannt, jedoch erst später von den Anhängern liberaler Denkschulen in ein Konzept gegossen, als diese sich mit dem, aus ihrer Sicht, „Scheitern« der Französischen Revolution auseinandersetzten. Unter diesen Denkern, die den Konstitutionalismus in Europa erneuerten, ragt Benjamin Constant besonders hervor. Er war der erste, der die Kluft zwischen der normativen Vorstellung von Verfassung und dem politischen Konstitutionalismus erkannte.

21 Constant geht davon aus, dass in der Gegenwart seiner Zeit Freiheit im ungestörten Genuss der privaten Autonomie besteht. Daraus folgt, dass einer der Zwecke einer guten Verfassung darin liegen müsse, den Genuss dieser persönlichen Freiheit sicherzustellen. Diese Garantie besteht in nichts anderem als dem Schutz gegen die stets mögliche Willkür der Regierenden. Die Verfassung ist, so Constant, »ein Akt des Misstrauens, da sie der Macht Grenzen aufzeigt«. Er sieht aber auch die Gefahr, die in einer neutralen Verfassung liegen könnte: »Der modernen Freiheit droht die Gefahr, dass wir, die wir im Genuss unserer persönlichen Unabhängigkeit und in der Verfolgung unserer privaten Angelegenheiten aufgehen, allzu billig unser Recht auf Anteil an der politischen Macht hergeben. ${ }^{15}$ Infolgedessen bestehe der andere Zweck einer Verfassung darin, die Teilhabe am politischen Leben zu gestalten, durch die Anerkennung des Wahlrechts, der Pressefreiheit und der Versammlungsfreiheit. Aus diesem doppelten Grundprinzip politikphilosophischer Natur ergeben sich mehrere Schlussfolgerungen in Bezug auf den Verfassungsbegriff.

Die erste betrifft das Wesen der Verfassung: Sie wird als rein anerkennender und deklaratorischer Akt verstanden und nicht als Gründungsakt, wie ihn die positivistische Denkschule sieht. Sie proklamiert lediglich Rechte des Einzelnen, die bereits vor der Gesellschaft bestanden, denn, so Constant, „der Mensch besitzt Rechte, die unabhängig von jedem Zusammenschluss bestehen «. ${ }^{16}$ Die zweite dieser Folgerungen lautet, dass der alleinige Inhalt der Verfassung das Bündel der »Freiheitsgrundsätze« ist, d.h. die Gesamtheit von Verfassungsgrundsätzen. Zu diesen Grundsätzen, die dem Schutz der Freiheit dienen, zählen sowohl die Pressefreiheit, die Verantwortlichkeit der Minister und der diesen untergeordneten Beamten und eine vielköpfige und unabhängige Volksvertretung ${ }^{17}$ als auch »die Gewähr, nicht willkürlich in Haft genommen zu werden, nicht seinem natürlichen Richter entzogen zu werden, keinen rückwirkenden Gesetzen unterworfen $\mathrm{zu}$ werden, sowie eine sehr geringe Anzahl weiterer Grundsätze ${ }^{18}{ }^{18}$ Die Aufzählung dieser Prinzipien ist nach Constants Ansicht im Übrigen nicht abgeschlossen, sie kann im Zuge der gesellschaftlichen Entwicklungen ergänzt werden, ganz im Sinne eines dynamischen Verfassungsverständnisses. Daraus ergibt sich, dass die Verfassung, wie Constant sie begreift, nicht mehr bloß als Verfassung im formalen Sinne verstanden wird, als schriftlich niedergelegtes, besonderes Gesetz. Constant schlägt eine materiell-rechtliche Definition der Verfassung vor, die man beispielsweise aus der Beschreibung von Verfassungsverstößen herauslesen kann. ${ }^{19}$ Diese Vorstellung wird durch Constants berühmten Ausdruck vollkommen zusammengefasst: „Die Verfassung ist die Versicherung eines Volkes: daher ist alles verfassungsmäßig, was sich auf die Freiheit bezieht, und ebenso kann daher nichts, was sich nicht auf die Freiheit bezieht, verfassungsmäßig sein. ${ }^{20}$ Mit anderen Worten, bestimmte Grundsätze werden selbst dann Verfassungsgrundsätze sein, wenn sie nicht in einem förmlichen Verfassungsgesetz festgeschrieben sind. Umgekehrt kann eine förmliche Verfassung 
(der Verfassungstext) Bestimmungen enthalten, die lediglich "regulatorischen« oder "verwaltungstechnischen " Charakter haben und damit nicht im materiellen Sinne Verfassungsnormen sind; es wäre ein Fehler, diese Bestimmungen "unterschiedslos als heilig " zu betrachten.$^{21}$ Eine solche Auslegung ist durchaus aktuell, denkt man an die Rechtsprechung des Verfassungsrates (Conseil constitutionnel) und anderer Gerichte über die wesentlichen, von den Gesetzen der Republik anerkannten Grundsätze Verfassungsgrundsätze, die aus formal einfachen Gesetzen abgeleitet wurden.

Schließlich besteht die letzte Schlussfolgerung darin, in der Verfassung auch eine Reihe von Mechanismen zum Schutz dieser Grundsätze zu sehen. Constant betont die Bedeutung von Verfahren und "Formen, diesen Hilfsgöttinnen menschlicher Zusammenschlüsse«. Mit heutigen Worten ausgedrückt könnte man sagen, dass Constant sich um die Wirksamkeit der freiheitlichen Verfassungsordnung Gedanken macht, denn er hat die Lehren aus der Französischen Revolution gezogen, während welcher, obgleich eine Verfassung die persönliche Freiheit verbürgte, »die Freiheit der Person ohne Unterlass missachtet wurde ${ }^{22} \mathrm{Er}$ sucht nach Wegen, wie derartige Verbrechen oder Verstöße gegen die Verfassung verhindert werden können, und schlägt daher eine Reihe von Maßnahmen und Einrichtungen zum besseren Schutz der Verfassungsgrundsätze vor. Die bekannteste und interessanteste dieser Einrichtungen, mit der Constants Name verbunden bleiben sollte, ist die berühmte "neutrale Gewalt" (pouvoir neutre) oder auch "bewahrende Gewalt" (pouvoir préservateur). Diese Schiedsinstanz ergibt sich für Constant folgerichtig aus den Erfordernissen, die sich aus der Logik des Verfassungssystems ergeben. Im Gegensatz zur monarchischen Regierungsform, in der es nur eine einzige Gewalt gibt und somit keine Konfliktmöglichkeiten bestehen, ergibt sich aus der Koexistenz aktiver und gleicher Gewalten in liberalen Verfassungsordnungen die Gefahr von politischen Auseinandersetzungen. Zwischen den Gewalten kommt es unausweichlich zu einem Ringen, denn »die Kämpfe der Freiheit kommen nie von selbst zur Ruhe, und wenn man die Kombattanten nicht voneinander scheidet, stirbt am Ende die Freiheit selbst «. ${ }^{23}$ Infolgedessen tut eine "neutrale« Gewalt not, die als dritte "Autorität» (nicht: Gewalt) in der Lage ist, Konflikte zwischen den »aktiven Gewalten« (die gesetzgebende und die ausführende Gewalt) zu schlichten und die Verfassung gegen jene zu verteidigen, die sie bedrohen.

So setzt Benjamin Constant sein Vertrauen nicht mehr in ein "Verfassungsgesetzbuch" oder ein »Verfassungsgesetz«, um das Hauptproblem des Konstitutionalismus zu bewältigen. Sein Lösungsansatz liegt in der harmonischen Verbindung zwischen Verfassungsgrundsätzen und deren Bewahrung durch eine außerordentliche Instanz. Bedeutung erlangt sein Werk dadurch, dass es zeigt, wie ein liberaler Denker ein Bewusstsein für die Entzweiung zwischen der Verfassung als Rechtsnorm und dem Konstitutionalismus als Weg für die Begrenzung der Macht entwickelt.

\section{Die Trennung von Verfassung und Konstitutionalismus nach Hegel}

Im Gegensatz zu Constant lehnt der deutsche Philosoph nicht nur die Idee der Verfassung als Rechtsnorm, sondern auch den Gedanken des Konstitutionalismus ab und stützt seine Gedanken stattdessen auf einen »institutionellen« Verfassungsbegriff. Beide Denker, die auch Zeitgenossen waren, teilten ähnliche Gedanken, gingen allerdings von unterschiedlichen Fragestellungen aus. Im Zentrum von Constants politischem Denken steht die Suche nach einem wirksamen Schutzmechanismus für die 
freiheitliche Verfassung; Hegel hingegen wählt als Ausgangspunkt seiner verfassungsphilosophischen Betrachtungen den Zusammenbruch des vom modernen Reich Napoleons besiegten Heiligen Römischen Reiches deutscher Nation. In seiner Schrift Die Verfassung Deutschlands reflektiert Hegel über die Nachteile, die sich aus der fehlenden Staatlichkeit Deutschlands ergeben, und sinniert, einem neuen Machiavelli gleich, über Wege, Deutschland eine wirkliche Verfassung zu geben, d.h. ein solides Staatswesen. Als Reaktion auf den nachkant'schen Idealismus und den rationalistischen und überhistorischen Universalismus der Französischen Revolution will er die konstitutionalistische Sichtweise überwinden, die seiner Ansicht nach den Staat auf dem Altar der individuellen Freiheit opfere. Aus diesem Grund stellt er zwei zentrale Ideen des Konstitutionalismus in Frage, die Idee des rule of law und die in den Theorien des Gesellschaftsvertrags enthaltene Idee des Individualismus. Er grenzt sich von der herrschenden konstitutionalistischen Sichtweise ab und stellt der Konstitution im Sinne der französischen Revolutionsverfassungen (normatives Verständnis des Verfassungsbegriffes) den Begriff der Verfassung im Sinne einer »Organisation des Staates« (politische oder »institutionelle« Verfassung) entgegen.

Hegel will die politische Philosophie der Antike (zumindest von Aristoteles) erneuern, von der er den Kerngedanken übernimmt, das Gemeinwesen (die Polis) sei erstrangig und gehe seinen Mitgliedern (den Bürgern) vor. Dieser Standpunkt führt dazu, dass Hegels Verständnis des Verfassungsgedankens durch sein Staatsverständnis bestimmt wird. Mit den Worten Bobbios: »Der Staat als organisches Gebilde ist etwas Strukturiertes und das Prinzip der Verfassung liegt gerade in der Organisation des Staates $~^{24}$ Als Verfassung des Staates ist die politische Verfassung zwangsläufig eine Verfassung des Volkes, da der Staat dieses überpersönliche Gebilde ist, das aus dem Volk ein organisches Ganzes formt. Bei Hegel entspricht die Verfassung dem tatsächlichen Aufbau des politischen Gebildes und nicht seiner normativen Struktur. ${ }^{25}$ Sie materialisiert das abstrakte Gebilde, den Staat. Hegel will damit ausdrücken, dass die Verfassung die Struktur oder die Gesamtheit ausdifferenzierter Strukturen darstellt, die ein Volk auf die Ebene der Staatswerdung hebt, eine sittlich höherwertige Einheit. Anders ausgedrückt ist für Hegel die (politische) Verfassung die »Form« oder auch das Prinzip der Einheit des Staates.

Als Verfassung des Staates vereinigt sie beide Ausprägungen der Souveränität des Staates, die innere und die äußere. In ihrer nach innen gerichteten Gestalt ist die politische Verfassung die »Organisation des Staates und der Prozeß seines organischen Lebens in Beziehung auf sich selbst « $^{26}$ und erschöpft sich nicht darin, eine Summe von Begrenzungen der Handlungen des Staates oder "Hindernis« für dieses Handeln oder ein Akt des Misstrauens gegenüber den Regierenden zu sein. ${ }^{27}$ Für Hegel gilt, »bei einer Verfassung kommt es [darauf] an [...] daß die besonderen Gewalten sich unterscheiden [...] aber ebenso in ihrer Freiheit zu einem Zweck zusammenarbeiten und von ihm gehalten werden, d. i. ein organisches Ganzes bilden. ${ }^{28}$ Damit sind die beiden der inneren Verfassung des Volkes eigenen Erfordernisse genannt, nämlich dessen Gliederung und Integrierung. Die Verfassung spiegelt die Gliederung der verschiedenen gesellschaftlichen Gruppen wider - sowohl die Unterscheidung zwischen Herrschenden und Beherrschten als auch die Aufteilung der verschiedenen Gewalten zwischen den verschiedenen gesellschaftlichen Gruppen. Die Verfassung legt die gesellschaftliche Spaltung offen, ist gleichzeitig aber auch das Vehikel, diese Gruppen zusammenzuführen. Sie vereint zwangsläufig voneinander getrennte Gruppen, deren 
Spaltung, bestünde sie fort, die Einheit des Staates unterhöhlen könnte. Weit davon entfernt, ein Instrument zur Sicherung der individuellen Freiheit zu sein, drückt die Verfassung den Grundsatz der Einheit des sozialen Gebildes aus, oder auch das Mittel, mit dem die bürgerliche Gesellschaft (dieser »äußere Staat«) über sich hinauswächst und eine höhere Ebene des Staatswesens erreicht.

Die Verfassung besitzt freilich auch eine internationale (nach außen gerichtete) Dimension: Sie ist das Instrument, mittels dessen der Staat als »individuelles Subjekt « seine Wesensart bei seinem Aufeinandertreffen mit anderen, d.h. in seinen Beziehungen mit den auswärtigen Mächten, den anderen Staaten, manifestiert. Er ist »eine Individualität ausschließendes Eins, welches sich damit zu anderen verhält, seine Unterscheidung also nach außen kehrt « ${ }^{29}$ Die Verfassung ist Ausdruck der Differenzierung, die bei der Herausbildung der Individualität des Staates im Konzert heterogener politischer Einheiten, der Gesellschaft der Völker, am Werke ist. Wegen der unvermeidlichen Streitigkeiten mit anderen Staaten ist es daher von Bedeutung, dass das Volk seine Verbundenheit mit der Verfassung zum Ausdruck bringt. Daraus ergibt sich für den Einzelnen »die Pflicht [...], diese substantielle Individualität, die Unabhängigkeit und Souveränität des Staats zu erhalten «. ${ }^{30}$ Dieses wesensbestimmende Band mit der Gemeinschaft der Bürger (dem Volk) erklärt Hegels institutionelles Verständnis von Verfassung: Die Verfassung ist eine sittlich-politische Kategorie (wie der Staat) und gleichzeitig beschreibt sie eine sowohl historische als auch lebendige Wirklichkeit, die im Geist des Volkes begründet liegt. »Es ist noch späterhin zu zeigen, daß die Verfassung eines Volkes mit seiner Religion, mit seiner Kunst und Philosophie oder wenigstens mit seinen Vorstellungen und Gedanken, seiner Bildung überhaupt [...] eine Substanz, einen Geist ausmache. ${ }^{31}$

Daraus ergeben sich bedeutende Veränderungen gegenüber dem Verfassungsverständnis der Revolutionszeit (dem Konstitutionalismus). Zunächst führt die Betonung des Geistes des Volkes als Schlüsselelement für das Vorhandensein einer Verfassung Hegel dazu, die Existenz einer verfassungsgebenden Gewalt zu leugnen. ${ }^{32}$ Des Weiteren führt diese Vorstellung dazu, in der Verfassung eher eine Beschreibung dessen zu sehen, was existiert, und nicht eine Anordnung dessen, was existieren soll. Sie spiegelt die tatsächlichen Empfindungen eines Volkes wider und nicht eine Bestimmung dessen, was es empfinden sollte. Mit anderen Worten, wird sie als das einem Volk je eigene betrachtet, dessen "Geist" in ihr zum Ausdruck kommen soll. Indem er postuliert, "Jedes Volk hat [...] die Verfassung, die ihm angemessen ist und für dasselbe gehört «, ${ }^{33}$ folgt Hegel dem Vorbild von Aristoteles und Montesquieu. Die Verfassung ist also weniger eine Norm (oder eine Gesamtheit von Normen) zur Begrenzung der Macht der Regierenden als Ausdruck der Eintracht zwischen dem Staat und den Mitgliedern der politischen Gemeinschaft. So hängt denn auch die Wirksamkeit einer Verfassung nicht so sehr davon ab, dass die (Rechts-)Normen der Verfassung vom Staat zum verbindlichen Gesetz erhoben werden, sondern vielmehr von der Anerkennung durch das Volk. Eine Verfassung ist wirklich, weil sie dem Geiste des Volkes entspricht.

\section{Das Erbe Hegels}

Wie bekannt, wurde das Hegelsche Verfassungsverständnis, in dessen Zentrum die politische Realität, und nicht Rechtsnormen, steht, in Deutschland von linken wie rechten Hegelianern aufgegriffen und fortgeführt. Im linken Spektrum (Marx und die 
Marxisten) hat Ferdinand Lassalle in einem berühmt gewordenen Vortrag die Frage nach dem "Verfassungswesen « aufgeworfen. Dieses Wesen wird von ihm als eine Kraft definiert, die den übrigen Gesetzen den Geist der Nation einhauche, gleichzeitig aber auch als unbestreitbares Prinzip gesehen, dessen Existenz aus Notwendigkeit und nicht aus Zufälligkeit entstanden sei. Diese Notwendigkeit einer Verfassung wird nunmehr mit marxistischen Begriffen umschrieben: sie ist der Ausdruck »der tatsächlichen Machtverhältnisse, die in einer gegebenen Gesellschaft bestehen «, mit anderen Worten innerhalb der Nation. Aus dieser den engen Einklang zwischen politischem Sein und Sollen bezeugenden Notwendigkeit folgt eine Relativierung des Verfassungstextes, somit auch der Verfassungsnorm, die lediglich als bloße schriftliche Niederschrift der streitbeladenen politischen Wirklichkeit angesehen wird. Somit ist der Übergang von einer politischen Verfassung zu einer »rechtlichen Verfassung « nichts weiter als das Niederschreiben der "tatsächlichen Machtverhältnisse [...] auf ein Blatt Papier«. Die Niederschrift der Verfassung zementiert diese Machtverhältnisse, die damit zu »Recht [...] zu rechtlichen Einrichtungen « werden; »und wer dagegen angeht, wird bestraft «. ${ }^{34}$ Doch bleibt das geschriebene Verfassungsrecht dem ehernen Gesetz der politischen Notwendigkeiten unterworfen, die jederzeit zu einer Änderung der rechtlichen Verfassung führen können. Das marxistische Verfassungsverständnis, wie jedes institutionelle Rechtsverständnis, bezieht sich auf die Entwicklungen der Gesellschaft und konzentriert sich mehr auf die politische Wirklichkeit als auf die Normen des Rechts. Der marxistische Ansatz radikalisiert die Hegelsche Sicht und sieht die Verfassungsnorm ausschließlich von den wirtschaftlichen und sozialen Verhältnissen abhängig.

31 Im Gegensatz dazu radikalisieren die Rechtshegelianer die Hegelsche Dialektik in eine autoritäre Richtung. Indem sie die Verfassung nach Rudolf Smend als das allumfassende Gesetz für das politische Leben in einem Staat definieren, geben sie der politischen Ordnung (derjenigen des Staates) gegenüber dem Konstitutionalismus den Vorzug. Dieses institutionelle Verfassungsverständnis bestreitet entschieden die Stichhaltigkeit, bezogen auf die Verfassung, der vom Rechtspositivismus vorgenommenen Unterscheidung zwischen dem politischen Sein, das für die Politik erkennbar ist, und dem in der Verfassung als Norm formulierten Sollen. Dieses Verständnis geht von der Prämisse aus, die politische Realität sei integraler Bestandteil des Verfassungsgedankens. Carl Schmitt, Musterbeispiel dieser Denkrichtung, definiert die Verfassung als Ergebnis einer "politischen Entscheidung«. Diese Entscheidung einer verfassungsgebenden Gewalt - hat den Zweck, "Form und Art der politischen Einheit« des Volkes zu begründen. ${ }^{35}$ Kurz gesagt: Die Verfassung gründet den Staat. Wie zuvor bei Aristoteles, Montesquieu oder Hegel, kann diese Selbstbestimmung in Verfassungsfragen in einer Form der Staatsorganisation münden, die die Eigenheiten des Geistes oder der Geschichte eines Volkes widerspiegelt, ohne zwangsläufig eine liberale Verfassung zu sein.

Im Gegensatz zu Hegel, der bei seinem Versuch, den Individualismus dialektisch zu überwinden, stets bemüht war, liberale Denkansätze zu bewahren, radikalisiert Schmitt das Hegelsche Denken in eine autoritäre und gegenrevolutionäre Richtung. Die Herrschaft des Staates über den Einzelnen erfolgt weder mittelbar durch zwischengeschaltete Gewalten noch in einer durch eine echte Gewaltenteilung gemilderten Form, und darüber hinaus wird die bürgerliche Gesellschaft mit Anarchie gleichgesetzt. Des Weiteren verleugnet diese dezisionistische Vorstellung von 
Verfassung, welche die Höhepunkte des kollektiven Seins der Gruppe in den Vordergrund stellt - den Krieg oder die Kriegsgefahr -, vollständig den Konstitutionalismus, indem sie systematisch der Staatsräson gegenüber der Rechtsnorm den Vorrang gibt. Dort, wo Locke als wahrer Liberaler das "Prärogativ« einhegen und begrenzen, es »konstitutionalisieren« will, strebt Schmitt danach, dieses Vorrecht von jeder Begrenzung zu befreien, damit die reine Entscheidungsgewalt eines allmächtigen Herrschers sich ohne Beschränkungen in den Freiräumen des Verfassungsgeflechts entfalten kann.

\section{Verfassungslehre und Verfassungspraxis oder die Entfremdung von Verfassung und Konstitutionalismus}

\section{Die Trennung in der Verfassungspraxis} Volkes auf und deckt die Gefahr des demokratischen Cäsarismus auf, die in der
Ausübung des allgemeinen Wahlrechts durch das Volk angelegt ist. So erscheint das Verhältnis von Konstitutionalismus und tatsächlicher Souveränität des Volkes als konfliktbeladen. Die vom Konstitutionalismus erdachten Verfassungsmechanismen sollten die Idee umsetzen, nach der die Verfassung dazu diene, den Einzelnen (als Angehörigen der Nation) gegen die Gewalten des Staates zu schützen. Durch die Demokratie und die mit ihr einhergehende Einrichtung des allgemeinen Wahlrechts entsteht eine neue potenzielle Gefahr für die Freiheiten des Individuums: die plebiszitäre Gefahr. Diese Gefahr ist politischer und rechtlicher Art. Sie erscheint zunächst im Primat des Volkswillens, der sich im Hinblick auf die Verfassung immer wieder ändern kann, und besteht darin, dass die »allgemeine Wahl schwerer wiegt, als die Verfassung«, d. h. dass der Wille der gewählten Mehrheit sich über die Verfassung erheben könnte. Der mögliche Widerstreit zwischen Konstitutionalismus und Volkssouveränität kann vorrangig bezüglich der Frage ausbrechen, ob das Volk (dessen Mehrheit) an die geltende Verfassung gebunden ist oder ob es diese nach seinem Gutdünken aufheben oder ändern kann. Letztlich besteht die Kernfrage darin zu bestimmen, ob das Volk in verfassungsmäßiger Weise eine liberale oder auch eine autoritäre Verfassung verabschieden kann. Wenn das letztgenannte Verständnis gelten sollte, würde die Logik der Souveränität, verstanden im formalen Sinne einer Entscheidung der Mehrheit, die liberale Logik (des Konstitutionalismus) zerstören. Nach der Sichtweise des Konstitutionalismus sind im Moment der Gründung einer konstitutionellen Demokratie der Gestaltungsfreiheit der verfassungsgebenden Gewalt des Volkes Grenzen gesetzt.

Der Konflikt zwischen dem Willen des Volkes und den Bestimmungen der Verfassung geht jedoch über die nur gelegentlich erfolgende Ausübung der verfassungsgebenden Gewalt hinaus und ist vor allem alltagspolitischer Natur. In einer Demokratie besteht einer der wesentlichen Grundsätze des Konstitutionalismus im Pluralismus der parteipolitischen Landschaft. Was ist daher zu tun, damit die Regierung des Staates durch die politische Mehrheit - ob nun in einer parlamentarischen oder einer 
präsidialen Demokratie - nicht zu einer Denaturierung der Verfassung und zu einer Abschaffung dieses konstituierenden Prinzips, des Pluralismus, führt? Diesbezüglich fordert die politische Philosophie im Namen der "Gerechtigkeit der Verfassung", dass eine faire Beteiligung der politischen Parteien oder auch eine »Form des fairen Konkurrenzkampfes um politische Macht und Ämter « ${ }^{36}$ vorgesehen sein muss. Mit anderen Worten: der Grundsatz der politischen Teilhabe und der Grundsatz der politischen Repräsentation (sowie des Prinzips einer Volksvertretung) gehören zu den elementaren Grundsätzen des politischen Konstitutionalismus.

Um die Idee der Verfassung als Rechtsnorm fortzuschreiben und ihre Bedeutung im konstitutionalistischen Sinne zu bewahren, wurde das Verfassungsarsenal erweitert, sodass eine Verfassung nunmehr auch Bestimmungen zum Schutz der Minderheit gegen mögliche Übergriffe durch die Mehrheit (d.h. der Parteien der Minderheit gegenüber den Parteien der Mehrheit) enthält und rechtliche Vorrichtungen vorgesehen sind, die die Einhaltung dieser Bestimmungen sicherstellen sollen. Unter diesen Schutzmechanismen ist der typischste die gerichtliche Normenkontrolle, also die Überprüfung der Verfassungsmäßigkeit von Gesetzen, die aufgrund eines pluralistischen Demokratieverständnisses (H. Kelsen) dem Gedanken folgt, dass die Verfassung (Grundprinzipien des Konstitutionalismus) gegenüber dem Willen der politischen Mehrheit überwiegt. Von diesem Standpunkt aus betrachtet, ist die Möglichkeit, eine solche gerichtliche Normenkontrolle auszulösen, eine der rechtlichen »Waffen" zum Schutz der Parteien der politischen Minderheit vor der Gefahr einer möglichen Unterdrückung durch die Partei(en) der politischen Mehrheit. Richtig ist allerdings auch, dass der ausschließlich gerichtliche Schutz der Verfassung an Grenzen stößt, die im Wesen der Handlungsmöglichkeiten der Gerichte begründet sind, wie z. B. dass diese Möglichkeiten, so in Fällen, in denen Dringlichkeit herrscht, weniger wirkungsvoll sind als diejenigen, die der Politik zur Verfügung stehen.

Zusätzlich zur plebiszitären Gefahr tritt in einer Demokratie das drohende Unheil einer Tyrannei der Mehrheit im soziologischen Sinne Tocquevilles. Darunter wird die Bedrohung durch die Macht der Gesellschaft verstanden, die »Macht der öffentlichen Meinung", die in einer Demokratie eine ebenso gewichtige Rolle einnimmt wie die traditionellen Inhaber der Macht (die öffentlichen Gewalten). Tocqueville sah vor allem die Gefahr eines sozialen Konformitätsdrucks, der weniger sichtbar und damit für den Konstitutionalismus schwerer zu erfassen sei. Heutzutage stellt sich die Frage, ob die wahre Gefahr nicht in einem Abgleiten der Macht der öffentlichen Meinung in eine Tyrannei der öffentlichen Meinung liegt. Die öffentliche Meinung im liberalen Sinne, im Zuge des Kampfes für eine Regierung "durch den Wettstreit der Ideen« geboren, droht in der Gegenwart durch neue Ausdrucksmöglichkeiten von Meinungen pervertiert zu werden. Die Pressefreiheit war die Verkörperung dieses Gedankens einer Regierung »durch den Wettstreit der Ideen« und war gemeinsam mit dem Grundsatz der Repräsentation des Volkes Teil des Markenkerns des Konstitutionalismus. Doch mit der Entstehung und gegenwärtigen Dominanz der neuen Medien scheint diese Regierungsform in Gefahr, treten Gefühle und Leidenschaften an die Stelle der Vernunft. Nach mancher Ansicht reiche die Macht der Medien sogar bis zu einer Denaturierung der öffentlichen Meinung und begünstige die plebiszitärsten Formen der Demokratie. Die Bedeutung dieser Tatsache - die Verschiebung der Macht von der Meinung zu den Medien - sei umso gravierender, da sie $\mathrm{zu}$ einer weiteren Verschiebung innerhalb der Demokratie hinzutrete, nämlich derjenigen zugunsten der Exekutive. Unter der Last der Anforderungen an den modernen Staat, den 
"vorantreibenden Staat«, führt die Wandlung der parlamentarischen Demokratie hin zu einer exekutiven Demokratie gleichzeitig zu einer »Personalisierung der Macht«. Diese Wandlung der Umstände, in denen der öffentliche Diskurs geführt wird, führt tendenziell dazu, dass bestimmte Ausprägungen des Parlamentarismus und bestimmte klassische Strategien des Konstitutionalismus obsolet werden.

An eine weitere Grenze stößt der Konstitutionalismus durch die Entstehung des Sozialstaats, der dessen Fundament und Wirksamkeit erheblich erschüttert hat. Aus Sicht der gesellschaftspolitischen Geschichte markiert die liberale Verfassung die Geburtsstunde der Abgrenzung von Staat und Bürgergesellschaft (nicht im Hegelschen Sinne verstanden). Wenn sich dieser Verfassungstyp darauf beschränkt, die Staatsgewalt einzuhegen, so geschieht dies aus einem individualistisch-freiheitlichen Staatsverständnis heraus, wonach die Gesellschaft sich selbst regeln soll. Die Verfassung ist eines der Instrumente des liberalen Staates, die die Eigeninitiative der Bürger und eine klare Abgrenzung von öffentlichem Raum und privatem Lebensbereich ermöglichen. Nun wurden allerdings die außerhalb der Verfassung selbst liegenden Voraussetzungen durch die gesellschaftlichen Umwälzungen und die industrielle Revolution tiefgreifend verändert. Wie bereits von Hegel und Marx dargelegt, bedrohen die »ökonomischen Kräfte« viel stärker als die politische Macht die Freiheit und die Würde des Menschen, während die Logik der Ungleichheit, die der Freiheit im Sinne der Liberalen innewohnt, das Gleichgewicht zwischen Gleichheit und Freiheit sprengt. Mitte des 19. Jahrhunderts verändert die "soziale Frage» das Wesen des Staates, der nun nicht mehr bloß der Garant der persönlichen Freiheiten ist, sondern auch zum Sozialstaat mutiert, der den Benachteiligten der kapitalistischen und industrialisierten Gesellschaft Leistungen erbringt. Auf die Menschenrechte folgen die sozialen Rechte, die Ansprüche anderer Art formulieren, da sie vom Staat keine Unterlassung, sondern eine aktive Handlung einfordern.

Die Folgen dieser hier nur in ihren groben Zügen dargestellten fundamentalen gesellschaftlichen Entwicklung sind zweierlei Art. Zunächst ergibt sich eine Einbuße der Wirk- und Ordnungskraft der »liberalen« Verfassung - der Verfassung im Sinne des Konstitutionalismus -, da diese ihrem Wesen und ihren "Mitteln « nach hauptsächlich darauf ausgerichtet war, die politische Macht zu zügeln, und nicht, die wirtschaftliche und finanzielle Macht einzudämmen. In dem Maße jedoch, in dem diese Macht stärker wird, versagt die »klassische« Verfassung durch eine allzu große Ausblendung der Realität, denn sie erfasst mehr die vermeintliche Macht (die Rechtsordnung) und weniger die tatsächliche (alle Mächte in ihrer Gesamtheit). Darüber hinaus führt der wirtschaftliche Interventionismus dazu, früher im Wesentlichen als unzulässig oder nur stark eingeschränkt als möglich erachtete Eingriffe des Staates in den privaten Bereich nunmehr als fast gewöhnliche Begebenheit anzusehen, die keiner besonderen rechtlichen Garantien bedarf. Schließlich verliert die Verfassung durch diese "materielle« Ausdehnung der Aufgaben des Staates auch einen großen Teil ihrer regulierenden Funktion, da die Funktionsweise des Sozialstaats weniger auf dem Verfassungsrecht als auf dem Verwaltungsrecht beruht. ${ }^{37}$ Trotz der Bemühungen der Rechtslehre wie der Rechtsprechung, dieses Eindringen des Staates in eine Rechtssphäre, die früher als unantastbar galt, einzudämmen, ist dem Wesen der neuartigen Eingriffsformen durch den Sozialstaat inhärent, dass sie den Gestaltungsmöglichkeiten durch das Recht und die Verfassung Grenzen setzen. So sind beispielsweise die Handlungen des "Wohlfahrtsstaates" schwieriger rechtlich einzugrenzen als diejenigen des »Nachwächterstaates«. Dieser handelt in seiner 
Funktion als Bewahrer der Rechte seiner Bürger »retrospektiv und punktuell«, und seine Handlungen können von der Rechtsnorm mühelos erfasst werden, durch eine klassische Subsumtion des Einzelfalles unter die allgemeine Norm. Jener hingegen handelt in seiner Funktion als Erbringer einer sozialen Leistung "prospektiv und flächendeckend « ${ }^{38}$ und diese Art der Handlungen, die vor allem auf ein zu erreichendes Ziel ausgerichtet sind, ist der rechtlichen Subsumtionslogik teilweise entzogen.

Durch das Auftreten der sozialen Frage auf der politischen Bühne ergibt sich als zweite Folge eine Veränderung der Substanz moderner Verfassungen. Diese umfassen nunmehr auch neuartige soziale Rechte und Grundsätze, die »unserer Zeit besonders nötig" sind (Präambel der französischen Verfassung von 1946). Im Gegensatz zur liberalen Verfassung, deren Bestimmungen rein organisatorische Fragen regeln und "Abwehrrechte" formulieren (zum Schutz der Grundrechte soll der Staat bestimmte Handlungen unterlassen), besteht die sozialstaatliche Verfassung aus Bestimmungen, die "materielle Fragen und Fragen bezüglich einer Leistung« regeln sollen. Seit dieser Zeit taucht der Begriff der "programmatischen Verfassung" auf, um diese eine Richtung vorgebende oder auch »ideologische« Form der modernen Verfassung zu beschreiben. Jedoch stößt diese bedeutende Wandlung des Inhalts und der Funktion einer Verfassung auf rechtliche Hürden. Unter anderem führen die inhaltlichen Veränderungen der Verfassungen dazu, die Wirkungskraft des Verfassungstextes zu schmälern, der nunmehr gespickt ist mit Vorschriften, die den Anschein erwecken, Rechtsnormen zu sein, allerdings nur einen schwachen normativen Gehalt aufweisen. Im Ergebnis dieser Entwicklung verliert die Verfassung ihren Anspruch, die politischen Handlungen der Staatsgewalt umfassend zu regeln: sie büßt an Wirkungskraft und Erhabenheit ein. So mindert die Entstehung des Sozialstaates die Macht des Konstitutionalismus, indem sie die Verfassung um eine "nicht-konstitutionalistische« Aufgabe erweitert.

\section{Die antikonstitutionalistische Sichtweise des Verfassungspositivismus}

41 Die (Verfassungs-)Rechtslehre hat die durch die demokratische und die soziale Revolution entstandene neue politische und soziale Wirklichkeit nicht unberücksichtigt gelassen. Sie hat daraus allerdings den verhängnisvollen Schluss gezogen, sich auf eine rein rechtliche Erfassung des Verfassungsbegriffs zurückzuziehen, womit sie Verfassung und Konstitutionalismus voneinander getrennt hat. Die herrschende Lehre setzt Verfassung mit dem Verfassungstext gleich und definiert die Verfassung somit als eine »Rechtsnorm», die die Beziehungen zwischen den öffentlichen Gewalten sowie zwischen der öffentlichen Gewalt und dem Einzelnen regeln soll. Es liegt im Wesen eines solchen positivistischen Verfassungsverständnisses, dass es den Bereich des Rechts und den Bereich der Politik radikal voneinander scheidet. Auf der einen Seite stehen die Gesetze des Rechts, auf der anderen die Gesetze der Politik.

Im Ergebnis wird damit der Gedanke des Konstitutionalismus auf dem Altar des Rechts, bzw. der »Rechtsnorm«, geopfert. Besonders deutlich geschieht dies bei Hans Kelsen, der die Theorie des Rechts und der Verfassung am konsequentesten ausformuliert hat. In der Absicht, die Selbständigkeit der Rechtswissenschaft zu begründen, treibt er das positivistische Verständnis der Verfassung als »Gesetz« oder »Rechtsnorm« auf die 
Spitze. Kelsen unterscheidet zwischen der rein formalen Verfassung, einer feierlichen Urkunde bestehend aus Rechtsvorschriften, die durch ein dafür vorgesehenes besonderes Verfahren geändert werden können, und der materiellen Verfassung, die Bestimmungen für die Schaffung allgemeiner rechtlicher Vorschriften enthält. Nur im Rahmen dieses materiellen Verfassungsverständnisses sieht Kelsen, beinahe widerwillig, die Möglichkeit (nicht die Notwendigkeit), einen Katalog mit Bürger- oder Menschenrechten oder auch die Gewaltenteilung vorzusehen. Keine der beiden Definitionen berücksichtigt dabei die politische Funktion einer Verfassung, und beide sind Ausdruck eines formalen Verfassungsverständnisses, nach dem die Verfassung eine Rechtsnorm darstellt, deren Geltung auf einer hypothetisch-deduktiv feststellbaren Grundnorm beruht. Diese Grundnorm, die vom Betrachter der Rechtsordnung als transzendentallogische Voraussetzung gesehen wird, kann jedoch das "Gutdünken des Herrschers" als Gehalt haben und so den Regierenden eine Blankovollmacht ausstellen.

Eine solche positivistische Sichtweise steht damit dem Konstitutionalismus diametral entgegen, denn sie führt dazu, jede in einem Verfassungsgesetz festgehaltene und mit einem höheren rechtlichen "Rang" als die übrigen Gesetze versehene Rechtsform des Staates als Verfassung anzusehen. Dementsprechend kann jeder Staatschef eines autoritären Staates Bestimmungen zu Verfassungsbestimmungen erklären lassen, die beispielsweise ein rein irreführendes Glaubensbekenntnis verkünden oder offen autoritären (und damit weniger heuchlerischen) Charakter haben, z. B. wenn sie einer einzigen Partei das Monopol politischer Tätigkeit zusprechen (Art. 6 der Verfassung der UdSSR von 1977). Der Inhalt einer Verfassung ist damit im wahrsten Sinne des Wortes gleichgültig geworden. Pseudo-Verfassungen (»autoritäre« Verfassungen) können nicht mehr von liberalen Verfassungen unterschieden werden. Infolgedessen ist die Verfassung vom Konstitutionalismus abgekoppelt: Ursprünglich erdacht als Weg zur Eindämmung staatlicher Willkür, ist sie zum Ausdruck eben dieser Willkür geworden (oder besser gesagt, kann sie dies jederzeit werden). Somit drückt das formale Verständnis von Verfassung ein Abdriften des Verfassungsbegriffes aus, wie der von Sartori und auch von anderen aufgestellte und eingangs erwähnte Befund aufzeigt. Der Rechtspositivismus schweigt bezüglich der Frage des Telos der Verfassung, er opfert diese politische Frage der methodologischen Reinheit (Unterscheidung zwischen Tatsachenurteilen und Werturteilen) und vor allem der Abgeschlossenheit des Rechtssystems in sich selbst. Damit lässt der Rechtspositivismus die dem Konstitutionalismus zugrundeliegende Hauptfrage unbeantwortet: "Was ist die politische Funktion einer Verfassung? «39

Wie es scheint, muss sich der Gedanke des Konstitutionalismus heutzutage einer zweifachen Herausforderung stellen, einer intellektuellen und einer praktischen. Zunächst die rechtstheoretische Herausforderung: die moderne Verfassungs- und Politikwissenschaft muss sich stets der Schwierigkeit stellen, ein normatives Verständnis des Verfassungsbegriffes hinzunehmen. Um jedoch die Möglichkeit normativer Vorstellungen überhaupt denken zu können, muss vorher die objektive Komponente, die einer Verfassung inhärent ist, herausgearbeitet werden, ohne dabei in eine rein ethische oder axiologische (Naturrecht) Definition zu verfallen, die in andere Wissensbereiche fällt. Wie alle bedeutenden verfassungspolitischen Schriften zeigen, ist nur eine Analyse der Politikgeschichte und des politischen Lebens in der Lage, diese objektive Komponente herauszuarbeiten, die es ermöglicht, einerseits dem »Verfassungsnihilismus« der positivistischen Sichtweise und andererseits einer Art 
"Verfassungsmoral« - um nicht zu sagen der Sehnsucht nach der alten Ordnung, die in den meisten institutionellen Auffassungen von Verfassung mitschwingt - zu entfliehen. Der Konstitutionalismus muss allerdings ebenso eine praktische Herausforderung bewältigen: die Entstehung neuer Formen der Ausübung und der Ausgestaltung von Herrschaft, die sich aus dem Strukturwandel des öffentlichen Raumes ergeben. Die Herausforderung besteht darin, die konstitutionelle Demokratie mit den neuen Erscheinungsformen der Herrschaft - »exekutive Demokratie«, Personalisierung und "Mediatisierung" der Macht - zu vereinbaren, wenn die Mechanismen zum Schutz gegen die Staatsgewalt mit den Veränderungen eben dieser Gewalt Schritt halten sollen. Jedoch erscheint der Konstitutionalismus, der die politische Macht durch eine als Rechtsnorm verstandene Verfassung einhegen wollte, gegenüber diesen neuartigen Herrschaftsformen zum Teil als machtlos, obwohl er doch gerade auf der Ebene der Verfassung nach (sowohl rechtlichen als auch politischen) Lösungen suchen sollte, die Herrschaft und deren diffuse Ausdrucksformen einzuhegen. Vielleicht kommt gerade in dieser Unfähigkeit, neue Regeln oder Mittel zu erdenken, eine gewisse Krise des Konstitutionalismus zum Ausdruck.

\section{BIBLIOGRAPHIE}

Bobbio, N. (1980): »Sur la notion de constitution chez Hegel«, Annales de philosophie politique, 12 (Cahiers Vilfredo Pareto), S. 133-144.

Bolingbroke, H. St. J. (1809 [1733/34]): »A dissertation upon parties« in: ders.: The Works, London: Hansaid \& Sons, Bd. 3, S. 3-312.

Cicero (1956): Über den Staat, übers. von Walther Sontheimer, Stuttgart: Reclam.

Constant, B. (1946 [1820]): »Von der Freiheit des Altertums, verglichen mit der Freiheit der Gegenwart«, in: ders.: Freiheit, übers. von Walter Lüthi, Basel: Schwabe.

Constant, B. (1991): Fragments d'un ouvrage abandonné sur la possibilité d'une constitution républicaine dans un grand pays, Paris: Aubier.

Constant, B. (1872 [1815]): »Principes de politique«, in: ders.: Cours de politique constitutionnelle, Paris: Guillaumin.

Franklin, J. H. (1963): Jean Bodin and the Sixteenth-Century Revolution in the Methodology of Law and History, New York: Columbia University Press;

frz. Übers.: Jean Bodin et la naissance de la théorie absolutiste, Paris: PUF, 1993.

Friedrich, C. J. (1953): Der Verfassungsstaat der Neuzeit, Berlin / Göttingen / Heidelberg: Springer.

Grimm, D. (1991): „Entstehungs- und Wirkungsbedingungen des modernen Konstitutionalismus«, in: ders.: Die Zukunft der Verfassung, Frankfurt am Main: Suhrkamp, S. 31-66.

Hegel, G. W. F. (1968 [1837]): Die Vernunft in der Geschichte, hg. von Johannes Hoffmeister, Hamburg: Meiner. 
Hegel, G. W. F. (1970 [1821]): Grundlinien der Philosophie des Rechts, Werke in zwanzig Bänden, Bd. 7, Frankfurt am Main: Suhrkamp.

Hegel, G. W. F. (1970 [1840]): Vorlesungen über die Philosophie der Geschichte, Werke in zwanzig Bänden, Bd. 12, Frankfurt am Main: Suhrkamp.

Jaume, L. (1993): »Garantir les droits de l'homme«, Tocqueville, XIV (1), S. 49-65.

Kelsen, H. (1929): Vom Wesen und Wert der Demokratie, Tübingen: Mohr.

La Déclaration des droits de l'homme, Paris: Hachette, 1988.

Lassalle, F. (1923 [1862]): »Über Verfassungswesen«, in: ders.: Auswahl von Reden und Schriften, Berlin: Dietz, S. 63-93.

Locke, J. (1967 [1690]): Zwei Abhandlungen über die Regierung, übers. von Hans Jörn Hoffmann, Frankfurt am Main: Europäische Verlagsanstalt.

Luhmann, N. (1990): »Verfassung als evolutionäre Errungenschaft«, Rechtshistorisches Journal, 9, S. $175-220$.

Mac Ilwain, C. H. (1940): Constitutionalism Ancient and Modern, Ithaka: Cornell University Press.

Mohnhaupt, H. (1990): „Verfassung«, in: Brunner, O. / Conze, W. /Koselleck, R. (Hg.):

Geschichtliche Grundbegriffe. Historisches Lexikon zur politisch-sozialen Sprache in Deutschland, Bd. 6, Stuttgart: Klett-Cotta.

Paine, T. (1973 [1791]): Die Rechte des Menschen, in der zeitgenössischen Übertragung von D. M. Forkel, bearbeitet und eingeleitet von Theo Stemmler, Frankfurt am Main: Suhrkamp.

Rawls. J. (1975 [1971]): Eine Theorie der Gerechtigkeit, übers. von Hermann Vetter, Frankfurt am Main: Suhrkamp.

Rials, S. (1986): »La question constitutionelle en 1814-1815: dispersion des légitimités et convergence des techniques (Une grande étape du constitutionnalisme européen)«, Annales d'histoire des facultés de droit, 3, S. 168 ff.

Sartori, G. (1962): „Constitutionalism: a preliminary discussion«, American Political Science Review, 56 (4), S. 853-864.

Sartori, G. (1992 [1987]): Demokratietheorie, übers. von Hermann Vetter, Darmstadt: Wissenschaftliche Buchgesellschaft.

Schmitt, C. (1993 [1928]): Verfassungslehre, 8. Aufl., Berlin: Duncker \& Humblot.

Sieyès, E. (1968 [1789]): »Was ist der dritte Stand?«, in: ders.: Abhandlung über die Privilegien. Was ist der dritte Stand?, übers. von Engelbert Oelsner, rev. von Rolf Hellmut Foerster, Frankfurt am Main: Insel.

Stourzh, G. (1988): "Constitution: changing meanings of the term from the early 17th to the late $18^{\text {th }}$ century «, in: Ball, T. / Pocock, J. G. A. (Hg.): Conceptual Change and the Constitution, Lawrence: University Press of Kansas, S. $35 \mathrm{ff}$.

Stourzh, G. (1989): Wege zur Grundrechtsdemokratie, Wien / Köln: Böhlau.

\section{NOTES}

1. Sartori (1992 [1987]), S. 319.

2. Friedrich (1953), S. 26. 
3. Locke (1969 [1690]), § 162, S. 311.

4. Grimm (1991), S. 37.

5. Stourzh (1988).

6. Bolingbroke (1809 [1733/34]), S. 157.

7. Cicero (1956), 1. Buch, 45, S. 43.

8. Mohnhaupt (1990).

9. Paine (1973 [1791]), S. 86.

10. Stourzh (1988).

11. Sieyès (1968 [1789]), S. 108.

12. Sieyès (1968 [1789]), S. 108 [Übersetzung modifiziert, A.d.Ü.].

13. Jaume (1993).

14. Luhmann (1990), S. 175.

15. Constant (1946 [1820]), S. 57.

16. Constant (1991), VIII, 8.

17. Constant (1872 [1815]), Principes de politique, Kap. XVIII.

18. Constant, Des réactions politiques.

19. Constant (1991), VIII, 11.

20. Constant, Principes de politique, S. 111.

21. Constant (1991), VIII, 11.

22. Constant, Principes politiques, Kap. XVIII.

23. Constant (1991), VIII, 4.

24. Bobbio (1980), S. 135.

25. Hegel (1970 [1821]), § 271, S. $431 \mathrm{f}$.

26. Hegel (1970 [1821]), § 271, S. 431.

27. Hegel (1970 [1821]), § 272, Zusatz, S. $434 \mathrm{f}$.

28. Hegel (1968 [1837]), S. 147.

29. Hegel (1970 [1821]), § 271, S. 431.

30. Hegel (1970 [1821]), § 324, S. 491.

31. Hegel (1970 [1840]), S. 64 f.

32. Hegel (1970 [1821]), § 273, S. 439.

33. Hegel (1970 [1821]), § 274, S. 440.

34. Lassalle (1923), S. 66, 73.

35. Schmitt (1993), S. 21.

36. Rawls (1975 [1971]), S. 257.

37. Grimm (1991).

38. Grimm (1991), S. 64.

39. Friedrich (1953).

INDEX

Mots-clés : constitution, constitutionnalisme

Schlüsselwörter : Verfassung, Konstitutionalismus 


\section{AUTEURS}

\section{OLIVIER BEAUD}

Olivier Beaud ist Professor für öffentliches Recht an der Universität Paris 2 Panthéon-Assas. Nähere Informationen finden Sie hier. 